\title{
You swallowed what?
}

\section{David Rayburn ${ }^{\mathrm{a}}$ and Cory Showalter ${ }^{\mathrm{b}}$}

a Indiana University,EmergencyMedicine/PediatricsResidency,Indianapolis,IN,United States

b Indiana University,PediatricEmergencyMedicine,Indianapolis,IN,UnitedStates

\section{Visual case discussion}

A previously healthy 7-year-old boy presented to the Emergency Department with a chief complaint of chest pain after swallowing a foreign body. Earlier that day the patient had been playing with his mother's nail clippers, disassembled the clipper handle and placed it in his mouth, then accidentally aspirated the clipper handle. Other than mild diffuse chest pain, the patient had no other complaints, specifically no cough, dysphagia, and no shortness of breath. On physical examination the patient was breathing comfortably with no drooling, stridor, or wheezing. His vital signs were all reassuring. Chest x-ray demonstrated a metallic foreign body remarkably located in the left main stem bronchus. Pediatric general surgery was consulted and the patient was taken to the operating room where he underwent bronchoscopy for removal of the airway foreign body. The patient tolerated the procedure well and there were no complications ( Figs. 1, 2 and 3 ).

This is the author's manuscript of the article to be published in final edited form as: 
Figure 1. Nail clipper, clipper mechanism on top of the nail clipper was the aspirated foreign body measuring approximately $4.5 \mathrm{~cm}$.

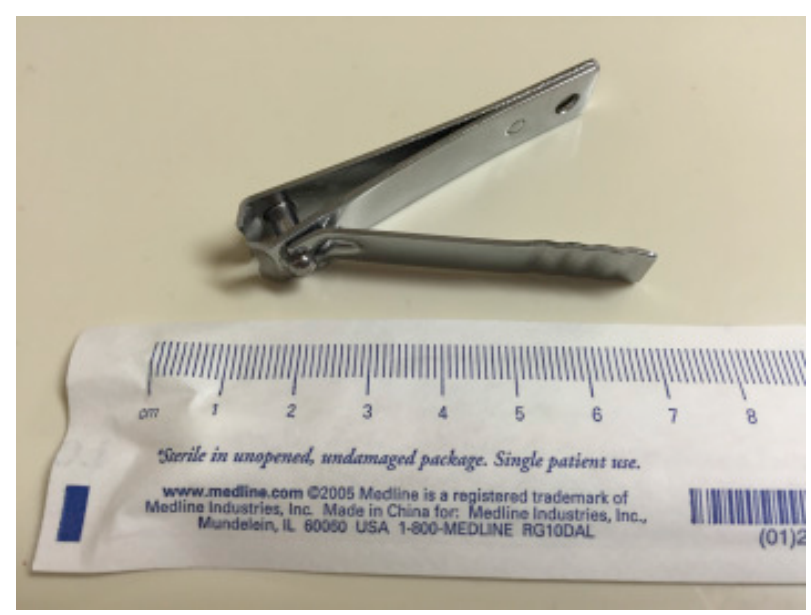


Figure 2. CXR PA view of foreign body in left main stem bronchus.

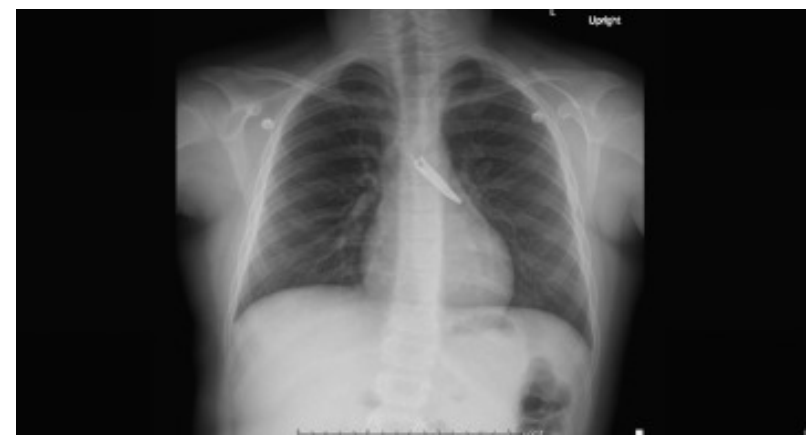


Figure 3. CXR Lateral view again demonstrating foreign body in the left main stem bronchus.

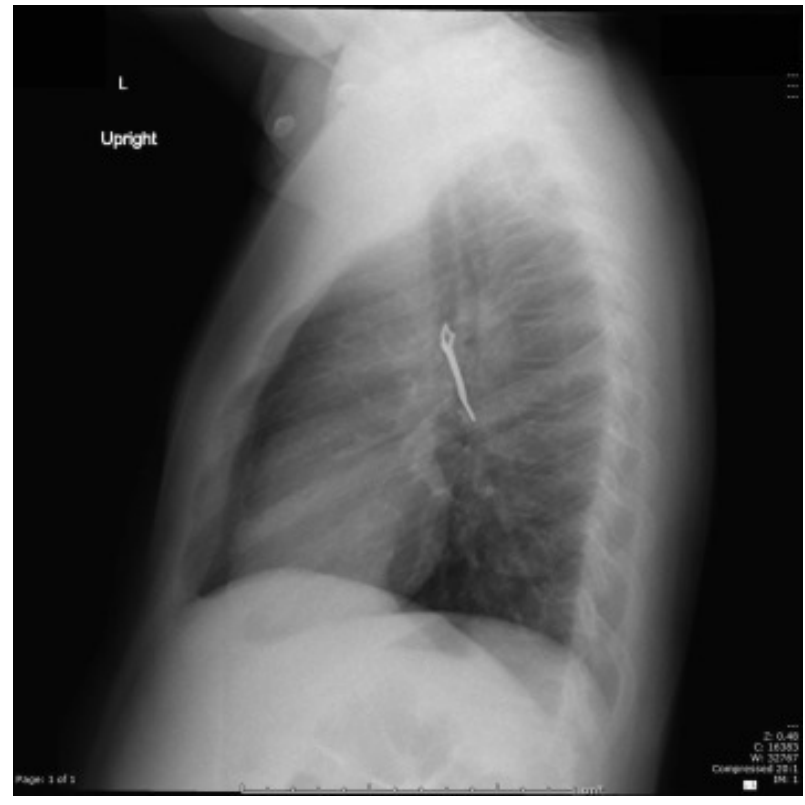

